\title{
A VIOLÊNCIA DOMÉSTICA INFANTOJUVENIL NA PERSPECTIVA DOS AGENTES COMUNITÁRIOS DE SAÚDE'
}

\author{
Carla Ferreira de Paula Gebara ${ }^{2}$ \\ Lélio Moura Lourenço \\ Telmo Mota Ronzani \\ Universidade Federal de Juiz de Fora, Juiz de Fora-MG, Brasil
}

\begin{abstract}
RESUMO. A presente pesquisa teve como objetivo analisar as crenças dos Agentes Comunitários de Saúde (ACSs) de um município de Minas Gerais a respeito da violência doméstica (VD) contra crianças e adolescentes. Participaram todos os trinta ACSs do município, os quais, num primeiro momento, responderam a um questionário autoaplicável, estruturado, individual e anônimo. Num segundo momento, os ACSs foram convidados a participar de um grupo focal. Os dados qualitativos foram analisados por meio da técnica de análise de conteúdo. Os dados indicaram uma forte tendência dos entrevistados a considerar o álcool e as drogas como geradores de VD contra crianças e adolescentes, assim como de problemas relacionais e intrafamiliares (falta de amor e carinho, falta de diálogo, falta de atenção e falta de uma estrutura familiar, por exemplo). Os resultados deixam clara a necessidade de se pensar em intervenções eficazes no plano institucional, visto que no espaço pesquisado não se desenvolve um projeto específico de prevenção e combate à VD, nem uma política voltada à capacitação dos ACSs para lidar com esta problemática.
\end{abstract}

Palavras-chave: Violência na família; grupos focais; crenças.

\section{DOMESTIC VIOLENCE AGAINST CHILDREN AND ADOLESCENTS IN THE PERSPECTIVE OF HEALTH COMMUNITY AGENTS}

\begin{abstract}
This research aims at analysing the beliefs of Health Community Agents (HCA) from a city of Minas Gerais, Brazil, regarding Domestic Violence (DV) against children and adolescents. Research participants were all 30 municipal HCA who, at first, answered a self-administered, structured, particular and anonymous questionnaire. Later on, the HCA were invited to participate in a Focus Group. Qualitative data were then analyzed using the content analysis technique. The data indicated a strong tendency of respondents to consider alcohol and drugs as generators of DV against children and adolescents, as well as intra-family and relationship problems (lack of love and affection, lack of dialogue, lack of attention and lack of a family structure, for example). These results make clear the need to think about effective interventions at the institutional level because, in that specific searched space, neither a specific project to prevent and combat DV nor a policy aimed at training the HCA to deal with this sort of problem were developed.
\end{abstract}

Key words: Family violence; focus groups; beliefs.

\section{LA VIOLENCIA DOMÉSTICA INFANTOJUVENIL EN LA PERSPECTIVA DE LOS AGENTES COMUNITARIOS DE SALUD}

RESUMEN. Este estudio tuvo como objetivo analizar las creencias de los Agentes Comunitarios de Salud (ACS) de un municipio de Minas Gerais sobre la violencia doméstica (VD) contra niños/adolescentes. Participaron todos los 30 ACS del municipio que, en un primer momento, respondieron a un cuestionario auto aplicable, estructurado, individual y anónimo. En segundo momento, los ACS fueron invitados a participar en un Grupo Focal. Los datos cualitativos fueron analizados mediante la técnica de análisis de contenido. Los datos indicaron una fuerte tendencia de los encuestados a considerar el alcohol y las drogas como generadores de VD contra niños/adolescentes, así como los problemas relacionales e intrafamiliares (falta de amor y afecto, falta de diálogo, falta de atención y falta de una estructura familiar, por ejemplo). Los resultados dejan claro que hay la necesidad de pensarse en intervenciones eficaces en el plano institucional, ya que, en el

\footnotetext{
1 Apoio e financiamento: Fundação de Amparo à Pesquisa do Estado de Minas Gerais (FAPEMIG).

2 Endereço para correspondência: Rua Antônio Altaf, 375/303. CEP 36.033-330. Juiz de Fora-MG, Brasil. E-mail: carla_gebara@yahoo.com.br
} 
espacio investigado no se desarrolla un proyecto específico para prevenir y combatir la VD y ni una política dirigida a la capacitación de los ACS para lidiar con esta problemática.

Palabras-clave: Violencia en la familia; grupos focales; creencias.

Compreende-se violência doméstica (VD) como todo ato ou omissão cometido por um membro da família em uma posição de poder, independentemente de onde ocorra, que prejudique $\mathrm{o}$ bem-estar físico, a integridade psicológica, a liberdade ou o direito ao desenvolvimento integral de outro membro da família (Shrader \& Sagot, 2000). Os atos violentos podem ser de natureza: física (envolve o uso da força física com a intenção de causar dano a outrem), psicológica (engloba agressões verbais ou gestuais, com a intenção de humilhar, ameaçar, causando danos à autoestima, por exemplo), sexual (abrange imposição de práticas de cunho sexual contra a vontade da pessoa ou que propiciem sua vitimização), ou ainda ser expressa na forma de negligência ou abandono, que envolve a ausência ou recusa de cuidados a quem necessita (Minayo, 2006).

Considerada como uma importante questão de saúde pública, a violência doméstica é, antes de tudo, uma violação dos direitos humanos, não escolhendo classe social, raça, credo, etnia, sexo ou idade. Embora esse tipo de violência ocorra em todas as faixas etárias, são as crianças e os adolescentes, por estarem em processo de crescimento e desenvolvimento, os que se apresentam em situação de maior vulnerabilidade e sofrem maiores repercussões para sua saúde (Sanchez \& Minayo, 2004).

Em crianças, a exposição direta ou indireta à violência doméstica está relacionada a um maior risco de consequências sociais e emocionais negativas. Condições familiares negativas e duradouras podem ser preditoras de baixo desempenho acadêmico, transtorno de estresse pós-traumático (PTSD), depressão, queixas somáticas, isolamento, ansiedade, agressividade, delinquência e uso de substâncias (Zanoti-Jeronymo, Laranjeira \& Figlie, 2008; WHO, 2009; Brasil, 2010; Moylan et al., 2010).

A intervenção nas situações de violência, especialmente da que acontece no âmbito familiar, tem sido tarefa destinada não só às esferas jurídica, policial e psicossocial: a identificação dessas situações, bem como de seus reflexos em termos de sofrimento e adoecimento, é questão que também se apresenta aos profissionais da área de saúde, que, nesse aspecto, carecem de preparo (Minayo, 2006). A prevenção da violência impõe grandes esforços, como, por exemplo, a superação das crenças de que se trata de um problema inevitável e exclusivo da área social. Tais problemas somente serão contornados quando se conhecer mais sistematicamente quem são esses profissionais e o que pensam, para que sejam propostas soluções que responsabilizem e criem rotinas nos serviços de saúde em relação ao tema.

Pelo fato de a atenção primária à saúde (APS) atingir a maior parte da população, considera-se este nível de atenção bastante estratégico para a detecção e implementação de práticas de prevenção à violência doméstica. Diante deste contexto, torna-se fundamental considerar os Agentes Comunitários de Saúde (ACSs), pois estes profissionais representam o elo entre o sistema de saúde e a comunidade onde vivem e trabalham, estabelecendo um contato mais próximo com as famílias do que os profissionais de cargos tradicionais, o que pode facilitar o trabalho de vigilância e promoção de saúde (Ronzani, Castro \& Formigoni, 2008; Lourenço, Cruvinel, Ameida \& Gebara, 2010).

Estudos nacionais apontam escassez e subnotificação de dados oficiais (conselhos tutelares, delegacias, etc.) a respeito da amplitude e das características da violência doméstica contra crianças e adolescentes (Brito, Zanetta, Mendonça, Barison \& Andrade, 2005; Costa et al., 2007; Bazon, 2008; Faleiros, Matias \& Bazon, 2009; Martins \& Jorge, 2009; Lourenço et al., 2010). Diante da lacuna de informações sobre o tema, torna-se necessário buscar outras fontes de informação. O presente trabalho buscou avaliar as crenças de Agentes Comunitários de Saúde (ACSs) de um município de pequeno porte da Zona da Mata mineira sobre o tema, numa tentativa de maior aproximação e conhecimento da realidade da violência doméstica, que ainda é bastante velada socialmente.

Considera-se que tais profissionais, atuando nas áreas de saúde primária, caracterizam-se como importantes sujeitos a serem analisados quanto às crenças sobre violência doméstica 
infantojuvenil, podendo ter ciência de casos "abafados" na própria família ou comunidade, que não chegam ao conhecimento de outros profissionais ou instituições (hospitais, delegacias, conselhos tutelares, escolas, creches, etc.). Sendo assim, acredita-se que o estudo das crenças dos ACSs possibilitará uma visão mais abrangente e realista do fenômeno da violência doméstica no universo investigado.

\section{MÉTODO}

\section{Participantes}

O presente estudo foi realizado em um município de pequeno porte da Zona da Mata de Minas Gerais, cujo nome não será mencionado por razões éticas. Em 2009, época em que os dados da pesquisa foram coletados, o município possuía cerca de 17.000 habitantes e quatro equipes do Programa de Saúde da Família (PSF). Participaram voluntariamente da pesquisa todos os trinta ACSs do município.

\section{Instrumentos}

$\mathrm{Na}$ pesquisa foram utilizados os instrumentos descritos abaixo.

- Questionário estruturado: para a coleta dos dados quantitativos, utilizou-se um questionário autoaplicável, estruturado, individual e anônimo, desenvolvido pelos autores e adaptado a partir de pesquisa realizada anteriormente (Gebara \& Lourenço, 2008). Tal questionário, composto por quinze questões fechadas, teve como objetivo averiguar as crenças dos ACSs a respeito da violência doméstica contra a criança e o adolescente, o tipo de violência doméstica mais frequentemente detectada, o grau de parentesco dos agressores, as providências tomadas diante de suspeita ou confirmação de ato violento e a correlação entre a violência doméstica e o consumo de álcool e/ou outras substâncias psicoativas.

- Entrevista coletiva com a utilização da técnica de grupo focal: para complementar as informações quantitativas e alcançar um maior aprofundamento do conhecimento das crenças e atitudes dos ACSs a respeito da violência doméstica contra crianças e adolescentes, foram realizadas entrevistas coletivas, utilizando-se a técnica qualitativa dos grupos focais (Patton, 2002).

\section{Cuidados éticos}

O projeto de pesquisa foi previamente submetido à apreciação do Comitê de Ética em Pesquisa da Pró-Reitoria de Pesquisa da Universidade Federal de Juiz de Fora, sendo aprovado em 20 de novembro de 2008, mediante o Parecer No 409/2008.

\section{Procedimentos para coleta de dados}

\section{Aplicação de questionário}

Para a aplicação dos questionários, aproveitou-se o espaço da reunião geral das equipes do PSF do município, a qual acontecia mensalmente e na qual a participação dos ACS era obrigatória. Foram necessárias duas reuniões para que todos os ACSs fossem entrevistados, e não houve nenhuma recusa em participar da pesquisa.

\section{Realização de grupo focal}

Foram realizados quatro grupos focais com os ACSs, que foram divididos de acordo com as equipes do Programa Saúde da Família (PSF) a que pertenciam. Os grupos serão chamados de $A, B, C$ e D. As entrevistas foram feitas no horário de trabalho, utilizando-se os espaços disponíveis nas próprias unidades, e foram combinadas com antecedência com cada equipe, de modo a não prejudicar o expediente dos ACSs. Desta forma, buscou-se uma maior aproximação com a realidade estudada, além de evitar o deslocamento dos profissionais do lócus de trabalho. Os grupos focais foram acompanhados por uma dupla de entrevistadores, um dos quais ficava livre para documentar as respostas, enquanto o outro coordenava a entrevista e o grupo, assumindo uma postura mais gerencial da técnica do grupo focal.

O roteiro dos grupos focais foi desenvolvido com base nas questões do questionário e complementado a partir das análises quantitativa e descritiva. As entrevistas foram gravadas em mídia eletrônica e posteriormente transcritas na integra.

\section{Procedimentos de análise de dados}

Os dados quantitativos provenientes dos questionários foram submetidos a análises estatísticas descritivas, considerando-se as 
frequências $(n)$ e as porcentagens relativas das respostas (\%). Todas as questões analisadas possuíam respostas categóricas, representando nas análises as seguintes variáveis qualitativas nominais: categorias profissionais, gênero, faixas de tempo de serviço em anos e um conjunto de crenças sobre diferentes aspectos da violência doméstica contra crianças e adolescentes.

A análise de dados dos grupos focais foi procedida por meio da técnica de análise de conteúdo do tipo estrutural e temática (Bardin, 2011), que consiste em descobrir os núcleos de sentido que compõem uma comunicação cuja presença ou frequência de aparição possam significar alguma coisa para o objetivo analítico visado. $\mathrm{Na}$ análise dos resultados qualitativos, visando à melhor organização e exploração dos resultados obtidos com os questionários e com as entrevistas em grupo focal, optou-se por seguir as seguintes etapas: pré-análise, exploração do material, tratamento dos resultados, inferência e interpretação (Bardin, 2011).

A primeira etapa da análise (pré-análise) consistiu na leitura flutuante para avaliação geral. Posteriormente, iniciou-se a exploração do material por meio da análise vertical dos dados, estabelecendo-se as categorias e subcategorias de acordo com os temas mais presentes. A análise vertical foi realizada por dois pesquisadores, e, em casos de divergência quanto à categorização das respostas, foi estabelecido um juiz com experiência em análise de conteúdo para se chegar a um consenso final. Como última etapa da análise, foi realizada a comparação e a horizontalização de cada entrevista dos grupos focais, para a visualização geral dos dados. Esta fase consistiu na junção e soma de frequência das categorias e subcategorias classificadas em cada resposta. Com base nos resultados das fases anteriores, passava-se para a inferência e interpretação a partir da fundamentação teórica e dos pressupostos norteadores da investigação.

$\mathrm{Na}$ análise dos grupos focais optou-se por priorizar a exploração qualitativa dos resultados, tomando-se cada grupo isolado como unidade diferente de análise e comparando os vários grupos entre si, destacando-se os pontos convergentes e divergentes entre eles. A comparação se concentrou nos tópicos mencionados, na variedade de crenças e atitudes para com esses tópicos entre os membros do grupo, nas etapas que a discussão examinou e nos resultados da discussão em cada grupo (Flick, 2009).

\section{RESULTADOS}

\section{Caracterização dos participantes}

Foram entrevistados todos os trinta Agentes Comunitários de Saúde (ACSs) do município, dos quais quatro $(13,3 \%)$ eram do sexo masculino e vinte e seis $(86,7 \%)$ eram do sexo feminino. A média de idade dos participantes foi de 33,3 anos. Em relação ao nível de escolaridade, $27(90 \%)$ agentes possuíam o Ensino Médio completo; dois (6,7\%) tinham o Ensino Fundamental completo, e apenas um $(3,3 \%)$ tinha curso superior.

No que diz respeito ao estado civil, quinze $(50 \%)$ agentes declararam-se casados; doze $(40 \%)$, solteiros; dois $(6,7 \%)$, viúvos e um $(3,3 \%)$, divorciado. De todos os respondentes, $64,3 \%$ declararam ter filhos. Destes, $26,7 \%$ tinham apenas um filho; $16,7 \%$ tinham dois filhos; $13,3 \%$ tinham três filhos e 3,3\% tinham cinco filhos. Foi possível observar certo equilíbrio em relação à área de atuação dos ACSs, visto que $56,7 \%$ deles atuavam na área urbana e $43,3 \%$ na área rural. A maioria dos entrevistados $(63,3 \%)$ tinha de um a cinco anos de profissão, $30 \%$ tinham de seis a dez anos, 3,3\% tinham menos de um ano e $3,3 \%$ tinham mais de dez anos. O tempo médio de atuação na instituição foi também de um a cinco anos para $63 \%$ dos agentes, de seis a dez anos para $33,3 \%$ deles e de menos de um ano para $3,7 \%$.

\section{Crenças sobre prevalência, causas e consequências da VD}

Nos grupos focais perguntou-se aos ACSs o que entendiam como violência doméstica contra crianças e adolescentes. As respostas refletiram tipos de violência e foram dadas, sobretudo, através de exemplos, visto que os ACSs fizeram uso de vários termos de seu vocabulário cotidiano e raramente deram nomes aos tipos de violência para ilustrar suas opiniões. Desta forma, termos como "falta de higiene", "sujeira" e "deixar a criança sozinha" foram interpretados como "negligência"; expressões como "desrespeito verbalmente", "agressão com palavras", "batendo psicologicamente" e "xingar" foram entendidas como "violência psicológica"; "bater" e "espancar" foram os termos mais frequentes para designar "violência física". Em 
todos os grupos houve destaque para a violência psicológica, que foi citada juntamente com a violência física, porém com maior frequência de exemplos. Os dados dos questionários também apontaram como ocorrências mais frequentes, na opinião dos ACSs, o "Abuso Psicológico", citado por $60 \%$, seguido do "Abuso Físico"(43,3\%) e da "Negligência" (33,3\%).

Com relação aos principais agressores, a "Mãe" foi apontada por todos os grupos, recebendo destaque nas equipes $B, C$ e D. $O$ "Pai" também foi citado em todos os grupos, mas recebeu mais ênfase apenas no grupo A. Esta crença foi frequentemente justificada pelos ACSs por argumentos como: "Eu acho que mais é a mãe. Ela fica com a criança o dia inteiro" (ACS 6, Grupo B). O "Padrasto" e a "Madrasta" foram identificados nos grupos A e B e os "Irmãos" citados apenas pelo grupo B. As respostas dos questionários foram semelhantes, visto que a mãe foi indicada por $63,3 \%$ dos respondentes como o principal agressor, seguida pelo pai $(60 \%)$ e pelo padrasto e madrasta ( $30 \%$ cada). Os irmãos foram apontados por $13,3 \%$ dos agentes, a categoria "Outros" foi citada por 3,3\% dos respondentes.

Outra questão abordada nos grupos focais foi a opinião dos ACSs sobre o que gera (motiva) a violência doméstica contra crianças e adolescentes. Em todos os grupos, os "problemas relacionais e intrafamiliares" - como a falta de amor e carinho, a falta de diálogo, a falta de atenção e a falta de uma estrutura familiar, por exemplo - foram predominantes. As questões socioeconômicas, como o desemprego e as dificuldades financeiras, também apareceram nos grupos A, B e C; da mesma forma, o uso de álcool/drogas foi citado pelos grupos A, C e D. O baixo nível educacional dos pais foi mencionado apenas pelos agentes do grupo C como fator gerador de violência doméstica contra crianças e adolescentes.

A relação entre a violência doméstica contra crianças e adolescentes com o uso de álcool e drogas foi destacada em todos os grupos focais, e não só como resposta a uma questão específica sobre o tema, pois surgiu em vários momentos diferentes da entrevista. Quando Ihes foi perguntado qual das substâncias psicoativas (álcool ou outras drogas) estaria mais relacionada aos casos de violência doméstica, suas respostas variaram de grupo para grupo. Nos grupos $A$ e $D$, a resposta predominante foi no sentido de apontar tanto o álcool quanto as drogas como igualmente envolvidos com as situações de violência; já os grupos $B$ e $C$ foram unânimes em apontar o álcool como mais fortemente relacionado aos episódios de violência doméstica. Vale ressaltar que em nenhum dos grupos foi dado destaque exclusivo às drogas ilícitas. Percebe-se que, no geral, os grupos focais deram um enfoque maior às bebidas alcoólicas como geradoras de violência, diferentemente das respostas individuais dos questionários, em que as drogas ilícitas apareceram como mais responsáveis por estimular a agressividade nos ambientes domésticos.

Foi possível observar ainda outros aspectos nos discursos dos grupos, como, por exemplo, as crenças sobre as consequências da violência doméstica para o desenvolvimento das crianças e adolescentes. A opinião mais frequente (surgiu em todos os grupos) foi que as crianças ou adolescentes que são vítimas de violência doméstica aprendem o comportamento agressivo com os pais e tendem a reproduzi-lo futuramente, seja como agressores dos próprios pais ou dos próprios filhos, no ambiente doméstico, seja com outras pessoas, em outros lugares (agredindo professores, por exemplo). "... E eles agridem o professor com as mesmas palavras que receberam em casa" (ACS5, Grupo A); "Porque hoje o pai bate no filho mas quando ele estiver com 15, 16 anos ele é que vai bater no pai!" (ACS1, Grupo D). Nesse contexto, vale ressaltar a crença dos ACSs no aspecto multigeracional da violência doméstica, pois nos quatro grupos focais foi apontada a relação entre a violência sofrida na infância/adolescência e a sua reprodução na fase adulta, sendo a violência doméstica um comportamento transmitido de geração para geração.

\section{Experiências pessoais e profissionais com VD}

Dos entrevistados, $75,9 \%$ afirmaram ter conhecimento sobre os diferentes tipos de violência doméstica contra crianças e adolescentes. Apenas onze agentes (36,7\%) afirmaram já ter detectado algum tipo de violência contra crianças e adolescentes atendidos ou visitados. Em todos os grupos focais foram comentados casos de VD detectados pelos ACSs em seu ambiente de trabalho. Os tipos mais relatados nesta questão foram o abuso físico (em três grupos), o abuso psicológico (em dois grupos) e a negligência (em um grupo), percebidos através de manifestações 
explícitas como gritos, agressões físicas (bater), marcas deixadas nos corpos e crianças trancadas em casa. Cabe ressaltar que em um dos grupos ficou evidente o despreparo dos ACSs para identificar casos de violência doméstica ("O meu (caso) eu não sei se é violência" - ACS2, Grupo D), o que indica a necessidade urgente de um treinamento específico nesta área.

A questão que apresentou maior divergência com relação às respostas do questionário foi a que dizia respeito às providências tomadas pelos ACSs diante da suspeita ou confirmação de casos de violência doméstica contra crianças e adolescentes. Nos grupos focais, o discurso coletivo revelou que a principal (e na maioria das vezes, única) providência tomada pelos agentes foi transmitir as informações para o(a) enfermeiro(a) chefe da equipe, que determina o melhor encaminhamento para cada caso. Vale ressaltar a diferença em relação às respostas individuais, do questionário, em que $90 \%$ dos agentes afirmaram fazer o encaminhamento ao Conselho Tutelar como principal providência em casos de violência contra crianças e adolescentes.

A relação dos ACSs com o Conselho Tutelar pareceu bastante tumultuada e ambígua, visto que muitos agentes disseram não confiar na eficácia do Conselho, por acreditarem que o órgão apresenta questões como falta de ética e de sigilo profissional ou por já terem tido experiências desagradáveis ao tentarem acionálo. Ainda sobre as providências tomadas, os grupos $A, B$ e $D$ admitiram não fazer nada diretamente com as famílias, e como principais dificuldades no gerenciamento dos casos apontaram a proximidade com as vítimas e agressores, já que são vizinhos dessas pessoas, pois residem na mesma comunidade em que trabalham. Segundo os ACSs, a atuação deles enquanto profissionais fica muito limitada diante dos casos de violência doméstica, porque fazem visitas mensais às mesmas residências e precisam estabelecer vínculos de confiança com as famílias que atendem:

... se eu sair daqui e for lá no Conselho Tutelar e denunciar uma pessoa, na próxima vez que eu for lá visitar aquela casa, a pessoa já vem com tipo: você veio aqui ontem e o hoje o Conselho Tutelar veio na minha casa! Quer dizer, a gente fica muito preso! Preso pra agir (ACS1, Grupo C)
Porque a gente necessita fazer a visita na família, e a base do nosso trabalho é a confiança! (ACS3, Grupo C). Se a gente perder a confiança da família, a gente perdeu tudo! E a gente não vai ter liberdade nem de chegar neles!A família não vai mais confiar na gente! (ACS5, Grupo C).

Além disso, outros obstáculos à prática efetiva dos ACSs no sentido de prevenir ou intervir nos casos de violência doméstica foram identificados, tais como as crenças na "palmada educativa", que surgiram em todos os grupos. Tais crenças estariam baseadas na ideia de que bater nas crianças seria uma forma (necessária e positiva) de educá-las em relação às regras e limites domésticos. Foram bastante frequentes os relatos dos agentes sobre experiências pessoais com violência doméstica, tanto como vítimas quando crianças, quanto como agressores dos filhos. Talvez por isto, muitos agentes relataram não se sentir confortáveis para orientar as famílias sobre esta questão, já que muitas vezes a violência doméstica está presente nas suas próprias vidas.

A última questão direcionada aos agentes pretendia averiguar se eles já haviam recebido algum tipo de treinamento para identificar e/ou intervir em casos de violência doméstica contra crianças e adolescentes. Os discursos de todos os grupos foram semelhantes, apontando a inexistência de um treinamento específico nesta área. Os ACSs acreditam que um treinamento específico seria de extrema importância, considerando-se as dificuldades encontradas pelos agentes na sua prática diária, não só em perceber os casos de violência doméstica, mas principalmente em realizar alguma intervenção direta com as famílias.

\section{DISCUSSÃO}

Com base nos resultados apresentados percebe-se que, tanto nas repostas dos questionários quanto nas discussões dos grupos focais, as crenças dos ACSs foram persistentes no sentido de apontar as mães como as principais agressoras das crianças e adolescentes. Os resultados de pesquisas nacionais (Brito et al., 2005; Costa et al., 2007; Assis, Avanci, Pesce, Pires \& Gomes, 2012) apontam a mesma tendência, sugerindo que a proximidade física e o tempo de permanência da 
mãe junto aos filhos, somados à maior responsabilidade culturalmente atribuída à mãe no processo educativo, parecem influenciar, de fato, o seu comportamento para com eles, favorecendo a ocorrência de situações de violência no ambiente doméstico. Além disso, é possível que a tendência de as mães aparecerem como as principais agressoras dos filhos sejam justificadas também em função do aumento do número de famílias monoparentais (Brandão \& Heilborn, 2006), em que, na maioria das vezes, os filhos acabam ficando sob os cuidados da genitora.

Em relação aos tipos de violência doméstica que ocorrem com maior frequência, os ACSs apontaram em primeiro lugar $\mathrm{o}$ abuso psicológico, seguido do abuso físico e da negligência. Um recente estudo (Assis et al., 2012) baseado nos registros do SINAN Sistema de Informação de Agravos de Notificação (VIVA Contínuo) - identificou 12.473 notificações de violência contra crianças no Brasil no ano de 2010, das quais a maior parte se referia a negligência/abandono, vindo a seguir a violência física. Outro estudo, realizado em Londrina, Paraná (Martins \& Jorge, 2009) a partir das notificações efetuadas em 2002 e 2006 aos conselhos tutelares e programas de atendimento a menores, identificou a violência física como a predominante, vindo em seguida a negligência/abandono. A pesquisa conduzida por Costa et al. (2007) nos conselhos tutelares de Feira de Santana - BA, encontrou como formas de violência prevalentes naquela região a negligência, seguida da violência física e da violência psicológica. Outro estudo, realizado no Centro Regional de Atenção aos Maus-Tratos na Infância (CRAMI), em Ribeirão Preto-SP, encontrou como forma de violência mais frequentemente notificada a violência física, seguida da negligência e da violência psicológica (Brito et al., 2005). A diferença quanto à ordem em que os tipos de violência foram apontados nas pesquisas pode estar relacionada a especificidades locais, refletindo a realidade sociocultural de cada contexto.

Não foi possível comparar as crenças dos ACSs sobre os tipos de violência doméstica mais frequentes com os dados epidemiológicos dos conselhos tutelares ou delegacias do município pesquisado, pois estas instituições não apresentavam um banco de dados estruturado sobre as ocorrências de violência doméstica contra crianças e adolescentes. A escassez de dados confiáveis sobre esta temática é um sério problema no Brasil e no mundo, tornando-se urgente a necessidade de maiores pesquisas na área, assim como a detecção e notificação de casos pelos serviços e profissionais de saúde (Assis et al., 2012); todavia, em vista da falta de dados estatísticos nesta área, a presente pesquisa mostra-se relevante, pois considera as crenças dos profissionais de saúde, que lidam diretamente com a questão da violência doméstica em seu cotidiano. Uma vez que tais crenças são construídas com base na experiência direta (Cavazza, 2008), acredita-se que o seu conhecimento permita uma maior aproximação da realidade (pelo menos no universo pesquisado) no que diz respeito à violência doméstica contra crianças e adolescentes.

O debate sobre a importância da notificação e a necessidade de tomá-la como ação desencadeadora de apoio institucional para as famílias ainda constitui uma grande tarefa: a de substituir a arraigada concepção de notificação como denúncia policial (Brasil, 2010). A baixa notificação nos serviços de saúde pode ser consequência do despreparo dos profissionais para lidar com as situações de violência e os encaminhamentos, seja pelos aspectos culturais que consideram a violência contra crianças e adolescentes como um problema exclusivo da família, seja pela falta de conhecimento das leis (Estatuto da Criança e do Adolescente), seja ainda pela obrigatoriedade de denúncias dos casos suspeitos (Costa et al., 2007; Bazon, 2008; Faleiros et al., 2009; Assis et al., 2012).

$\mathrm{Na}$ presente pesquisa ficou claro que os ACSs não se sentem preparados para identificar casos de violência doméstica, talvez porque nunca tenham recebido um treinamento específico nesta área. Além disso, nos grupos focais foi evidenciada a inexistência de qualquer atuação dos ACSs diante de situações de violência doméstica - seja pela notificação dos casos, seja por algum tipo de intervenção. Esses profissionais relataram ter medo de fazer a notificação, principalmente em função do provável comprometimento na comunidade onde vivem e trabalham. Foi possível observar crenças (individuais e coletivas) em relação à ineficácia e/ou incompetência dos órgãos jurídicos (especialmente o Conselho Tutelar), que também podem contribuir para a falta de denúncias, notificações ou encaminhamentos por parte dos ACSs. 
Por outro lado, eles também relataram não saber o que fazer em casos de violência doméstica contra crianças e adolescentes. Este dado é bastante preocupante, considerando-se a posição estratégica dos ACSs, cuja profissão vem na tentativa de aproximar a saúde a questões mais amplas, comunitárias, podendo ser ainda mais importante na questão da violência doméstica. Considera-se que tais profissionais, inseridos na equipe do PSF, tomam a família, e não o indivíduo, como a referência para as práticas de saúde, intervindo ativa e preventivamente, num enfoque comunitário (Ronzani et al., 2008).

Por outro lado, embora a equipe (composta por médicos, enfermeiros e agentes comunitários) esteja em contato próximo com as famílias, há ainda muitas dificuldades para lidar com a questão da violência nesse âmbito, exigindo-se uma formação orientada para esse problema (Brasil, 2010). Este aspecto também pôde ser constatado no presente estudo. Sendo assim, torna-se fundamental que os gestores elaborem políticas públicas de prevenção e combate à violência doméstica, assumindo a capacitação profissional como forma de promover uma atenção especializada e adequada à população.

Outro aspecto importante observado no presente trabalho refere-se ao peso dado pelos ACSs aos fatores relacionais e intrafamiliares como geradores de violência doméstica, os quais são mais destacados do que questões socioeconômicas. Foi interessante observar a crença dos ACSs - que na sua maioria apresentam baixo nível de escolaridade e renda (comparativamente aos outros profissionais de saúde) - de que o nível educacional e a situação financeira não estariam tão relacionados à violência doméstica quanto os aspectos afetivos e relacionais ou o uso de álcool e drogas. De certa forma, a associação entre pobreza e violência doméstica encontra-se respaldada por alguns estudos cujos resultados demonstram uma frequência de maus-tratos infantojuvenis $50 \%$ maior em famílias com pais desempregados (Reichenheim, Dias \& Moraes, 2006).

De fato, pesquisas têm mostrado que as más condições socioeconômicas constituem risco para violência doméstica, ainda que de forma moderada ou não linear (Reichenheim, Dias \& Moraes, 2006; Dossi, Saliba, Garbin \& Garbin, 2008; D'Oliveira et al., 2009). Sugere-se que o estresse relacionado às más condições de vida e à não garantia de acesso a bens e serviços constituiria a base dessa associação; no entanto, tal associação indiscriminada pode levar a uma criminalização da pobreza, colocando alguns segmentos sociais na mira de julgamentos prévios. É preciso considerar que a maior frequência de violência nas classes sociais menos favorecidas pode estar relacionada ao fato de que as pessoas mais pobres tendem a denunciar mais, dando visibilidade ao problema, geralmente ocultado entre os mais abastados economicamente (Dossi et al., 2008).

Já em um estudo transversal realizado com uma amostra representativa de mulheres na cidade de São Paulo e na Zona da Mata de Pernambuco para estimar a frequência e os fatores associados à violência doméstica em diferentes contextos socioculturais, os fatores socioeconômicos (assim como na presente pesquisa) foram relativizados, apresentando menor importância em comparação com outros, como escolaridade até oito anos, abuso sexual na infância, cinco ou mais gestações e problemas com a bebida (D'Oliveira et al., 2009). A escolaridade também mostra associação com violência doméstica em outros estudos, com proteção nos níveis mais altos e maior risco associado aos níveis mais baixos (Reichenheim, Dias \& Moraes, 2006; Dossi et al., 2008; D'Oliveira et al., 2009). Por outro lado, em concordância com as crenças dos ACSs, pesquisas apontam os laços afetivos e as relações familiares sadias como importantes fatores de proteção contra a violência doméstica infantojuvenil (Brito et al., 2005, Abramsky et al, 2011).

No presente estudo, também chamaram atenção as crenças dos ACSs a respeito das possíveis consequências da violência doméstica para o desenvolvimento das crianças e adolescentes. No geral, as consequências mais percebidas foram a futura agressividade das crianças/adolescentes vítimas, além de problemas psiquiátricos (como a depressão) e problemas na escola. As crenças dos ACSs vão ao encontro de outros estudos, que sugerem um padrão multigeracional da violência doméstica (White, McMullin, Swartout, Sechrist \& Gollehon, 2008; Martins \& Jorge, 2009). Existem evidências de que a exposição infantil à violência doméstica (seja como testemunhas ou como vítimas diretas), além do sofrimento associado, pode ser um fator de risco importante para a 
perpetuação do comportamento violento na vida adulta (Zanoti-Jeronymo et al., 2008; Martins \& Jorge, 2009). Além disso, mais uma vez respaldando as crenças dos ACSs, pesquisas apontam uma série de consequências sociais e emocionais negativas da violência doméstica para o desenvolvimento de crianças e adolescentes, entre elas: transtornos de ansiedade, depressão, distúrbios do sono, delinquência, gravidez precoce e uso de álcool e drogas (White et al., 2008; Zanoti-Jeronymo et al., 2008; WHO, 2009). Vale ressaltar que todos esses fatores foram citados pelos ACSs ao longo dos grupos focais.

Outra questão que obteve destaque nos grupos focais, apesar de não ter sido abordada diretamente pelos pesquisadores, foi a da "palmada educativa". Os ACSs apresentaram crenças de que o castigo físico representa um método aceitável e eficiente na educação e disciplina de crianças, e muitos deles reconheceram fazer uso deste meio para colocar limites nos próprios filhos. Não obstante, diferentes pesquisas elaboradas com o objetivo de relacionar o recebimento da punição corporal com alguma variável comportamental da criança encontraram prejuízos para o desenvolvimento infantil: as crianças que apanhavam (comparadas com as que não apanhavam) apresentavam autoestima mais baixa, comportamento mais agressivo, maior frequência de sintomas psiquiátricos e menor bem-estar geral (Lansford et al., 2009; Moylan et al., 2010). Ficou claro que muitos ACSs não consideram essa prática como uma forma de violência, pois até a recomendam como estratégia educativa. Sendo assim, é evidente que a capacidade de intervenção e atuação dos ACSs junto à comunidade fica bastante limitada em função das próprias crenças que eles têm a respeito da naturalização da violência doméstica. Diante disso, torna-se fundamental um trabalho educativo e informativo com esses profissionais, no sentido de tentar uma reconstrução cognitiva, sensibilizando-os para a questão da violência familiar.

Os resultados da presente pesquisa indicaram uma forte tendência de inserção do álcool e da droga no universo da violência doméstica, segundo as crenças dos respondentes. No Brasil, um levantamento domiciliar mostrou que os agressores estavam embriagados em $52,7 \%$ dos domicílios com histórico de violência, enquanto em 9,7\% dos casos o agressor estava intoxicado por outra droga (Noto, Fonseca, Silva \& Galduròz, 2004). Outros estudos também apontam a relação entre violência doméstica e uso de substâncias psicoativas, sugerindo que o consumo pelos pais pode aumentar os riscos de ocorrência de atos violentos contra os filhos. Além disso, crianças e adolescentes que experimentam situações de violência têm maior risco de desenvolver problemas futuros com uso de substâncias, perpetuando, assim, um contínuo e crescente ciclo de violência/abandono (Zilberman \& Blume, 2005; Zanoti-Jeronymo et al., 2008; WHO, 2009).

Embora existam fortes associações entre violência doméstica e consumo de álcool e outras drogas, ainda há muitas controvérsias sobre como essa relação acontece e são raros os estudos sobre as peculiaridades dessa associação, principalmente em países em desenvolvimento, como é o caso do Brasil. Pesquisas recorrentes sobre o tema se mostram necessárias, tendo em vista a importância das crenças dos profissionais de saúde observadas na presente pesquisa e a carência de uma literatura mais qualificada, ligada às especificidades do tema.

\section{CONSIDERAÇÕES FINAIS}

Desde as últimas décadas o tema da violência tem sido vinculado à área da saúde, apontando a necessidade de envolvimento e preparo dos profissionais e dos serviços de saúde, dada a conjuntura complexa e desafiante desse fenômeno. Nesse contexto, destaca-se o papel dos Agentes Comunitários de Saúde (ACSs) como importantes atores na execução de práticas preventivas, em face da posição estratégica que ocupam na comunidade.

Torna-se necessário que os serviços (postos, centros de saúde, hospitais, resgates) e os profissionais de todos os níveis e categorias reconheçam que devem se inserir no processo de prevenção da violência, na identificação de condições de risco e na atenção às vítimas. Para tanto, é preciso que questionem o conhecimento que têm do problema, assim como o preparo e a capacidade de intervenção diante dos comportamentos observados. A presente pesquisa permitiu constatar que, pelo menos no município pesquisado, não existe uma política voltada para a capacitação dos profissionais de 
saúde no que concerne à violência doméstica infantojuvenil. A questão da capacitação direcionada especificamente aos ACSs torna-se fundamental, já que pode afetar diretamente as crenças desses profissionais sobre o fenômeno diante da sua própria comunidade, podendo interferir diretamente nas suas atitudes e práticas relacionadas a esse problema.

Este estudo também deixou clara a necessidade de se pensar em intervenções eficazes no plano institucional, ressaltando que, no espaço pesquisado, de acordo com os resultados encontrados, não se desenvolve um projeto específico em relação à violência intrafamiliar. Esses resultados sugerem a elaboração e implantação de políticas públicas de prevenção e combate à violência doméstica infantojuvenil, beneficiando não só as instituições de apoio às vítimas do município pesquisado, mas também a sociedade e o poder público em geral, supostamente interessados em conhecer e estabelecer estratégias eficazes para lidar com essas situações.

\section{REFERÊNCIAS}

Abramsky, T., Watts, C. H., Garcia-Moreno, C., Devries, K., Kiss, L., Ellsberg, M., Jansen, H. A. F. M. \& Heise, L. (2011). What factors are associated with recent intimate partner violence? Findings from the WHO multi-country study on women's health and domestic violence. BMC Public Health, 11:109.

Assis, S. G., Avanci, J. Q., Pesce, R. P., Pires, T. O. \& Gomes, D. L. (2012). Notificações de violência doméstica, sexual e outras violências contra crianças no Brasil. Ciência \& Saúde Coletiva, 17(9):2305-231.

Bardin, L.(2011). Análise de Conteúdo. Lisboa: Edições 70.

Bazon, M. R. (2008). Violências contra crianças e adolescentes: análise de quatro anos de notificações feitas ao Conselho Tutelar na cidade de Ribeirão Preto, São Paulo, Brasil. Cadernos de Saúde Pública, 24(2), 323-332.

Berlin, L. J., Malone, P. S., Ayoub, C., Ispa, J. M., Fine, M, A., Brooks-Gun, J. et.al. (2009). Correlates and Consequences of Spanking and Verbal Punishment for Low-Income White, African American, and Mexican American Toddlers. Child Development, 80(5), 1403-1420.

Brandão, E. R \& Heilborn, M. L. (2006). Sexualidade e gravidez na adolescência entre jovens de camadas médias do Rio de Janeiro, Brasil. Cadernos de Saúde Pública, 22(7), 1421-143.
Brasil. Ministério da Saúde, Secretaria de Atenção à Saúde, Departamento de Ações Programáticas e Estratégicas. (2010). Linha de cuidado para a atenção integral à saúde de crianças, adolescentes e suas famílias em situação de violência: orientações para gestores e profissionais de saúde. Brasília: Ministério da Saúde.

Brito, A. M., Zanetta, D. M. T., Mendonça, R. C. V., Barison, S. Z. P., \& Andrade, V. A. G. (2005). Violência doméstica contra crianças e adolescentes: estudo de um programa de intervenção. Ciência \& Saúde Coletiva, 10(1), 143-149.

Cavazza, N. (2008). Psicologia das atitudes e das opiniões. São Paulo: Edições Loyola.

Costa, M. C. O., Carvalho, R. C., Santa Bárbara, J. F. R., Santos, C. A. S. T., Gomes, W. A., \& Sousa, H. L. (2007). O perfil da violência contra crianças e adolescentes, segundo registros de Conselhos Tutelares: vítimas, agressores e manifestações de violência. Ciência \& Saúde Coletiva, 12(5), 1129-1141.

D'Oliveira, A. F. P. L., Schraiber, L. B., França-Junior, I., Ludermir, A. B., Portela, A. P., Diniz, C. S., et al. (2009). Fatores associados à violência por parceiro íntimo em mulheres brasileiras. Revista de Saúde Pública, 43(2), 299-310.

Dossi, A. P., Saliba, O., Garbin, C. A. S. \& Garbin, A. J. I. (2008). Perfil epidemiológico da violência física intrafamiliar: agressões denunciadas em um município do Estado de São Paulo, Brasil, entre 2001 e 2005. Cadernos de Saúde Pública, 24(8), 1939-1952.

Faleiros, J. M., Matias, A. S. A. \& Bazon, M. R. (2009). Violência contra crianças na cidade de Ribeirão Preto, São Paulo, Brasil: a prevalência dos maus-tratos calculada com base em informações do setor educacional. Cadernos de Saúde Pública, 25(2), 337-348.

Flick, U. (2009). Introdução à pesquisa qualitativa. Porto Alegre: Artmed.

Gebara, C. F. P. \& Lourenço, L. M. (2008). Crenças de Profissionais da Saúde sobre Violência Doméstica contra Crianças e Adolescentes. Psicologia em Pesquisa, 2(01), 27-39.

Lansford, J. E., Dodge, K. A., Pettit, G. S., Criss, M. M., Shaw, D. S. \& Bates, J. E. (2009). Trajectories of Physical Discipline: Early Childhood Antecedents and Developmental Outcomes. Child Development, 80(5), 1385-1402.

Lourenço, L. M., Cruvinel, E., Almeida, A. A. \& Gebara, C. F. P. (2010). Estudo das Crenças dos Agentes de Saúde a Respeito da Violência Doméstica. Estudos Interdisciplinares em Psicologia, 1(1), 108-128.

Martins, C. B. G. \& Jorge, M. H. P. M. (2009). A violência contra crianças e adolescentes: características epidemiológicas dos casos notificados aos Conselhos Tutelares e programas de atendimento em município do Sul do Brasil, 2002 e 2006. Epidemiologia e Serviços de Saúde, 18 (4), 315-334. 
Minayo, M. C. S.(2006). Violência e Saúde. Rio de Janeiro: Fiocruz.

Moylan, C. A., Herrenkohl, T. I., Sousa, C., Tajima, E.A., Herrenkohl, R. C. \& Russo, M. J. (2010). The effects of child abuse and exposure to domestic violence on adolescent internalizing and externalizing behavior problems. Journal of Family Violence, 25, 53-63.

Noto, A. R., Fonseca, A. M., Silva, E. A., \& Galduròz, J. C. F. (2004). Violência Domiciliar Associada ao Consumo de Bebidas Alcoólicas e de Outras Drogas: Um Levantamento No Estado De São Paulo. Jornal Brasileiro de Dependência Química, 5(1), 9-17.

Patton, M. Q. (2002). Qualitative Evaluation and Research Methods. London: SAGE.

Reichenheim, M. E., Dias, A. S., \& Moraes, C. L. (2006). Co-ocorrência de violência física conjugal e contra filhos em serviços de saúde. Revista de Saúde Pública, 40(4), 595-603.

Ronzani, T. M., Castro, P. M. \& Formigoni, M. L. O. (2008). Avaliação de um processo de implementação de práticas de prevenção ao uso de risco de álcool entre agentes comunitários de saúde. HU Revista, 34(1), 9-18.

Sanchez, R. N., \& Minayo, M. C. S. (2004). Violência contra Crianças e Adolescentes: Questão Histórica, Social e de Saúde. In C. A. Lima (Org). Violência faz mal à saúde (pp. 23-8). Brasília: Ministério da Saúde.
Shrader, E., \& Sagot, M. (2000). Domestic violence: women's way out. Pan American Health Organization. Washinton: States Unites of America.

White, J. W., McMullin, D., Swartout, K., Sechrist, S., \& Gollehon, A. (2008). Violence in intimate relationships: A conceptual and empirical examination of sexual and physical aggression. Children and Youth Services Review, 30, 338351.

World Health Organization (WHO). (2009). Violence prevention: the evidence. Geneve: WHO. 127p.

Zanoti-Jeronymo, D. V., Laranjeira, R., \& Figlie, N. B. (2008). Efeitos do abuso do álcool relacionados à violência doméstica nos filhos: um levantamento bibliográfico. Revista Brasileira de Psiquiatria, 30(2), 168-76.

Zilberman, M.L., \& Blume, S.B. (2005). Domestic violence, alcohol and substance abuse. Revista Brasileira de Psiquiatria. 27(supl 2), S51-5.

Recebido em 13/05/2012 Aceito em 26/04/2013

Carla Ferreira de Paula Gebara: mestre em Psicologia pela Universidade Federal de Juiz de Fora, doutoranda em Psicobiologia pela Universidade Federal de São Paulo, Brasil. Pesquisadora colaboradora do Núcleo de Estudos em Violência e Ansiedade Social (NEVAS) do Departamento de Psicologia da Universidade Federal de Juiz de Fora, Brasil.

Lelio Moura Lourenço: doutor em Psicologia Social pela Pontifícia Universidade Católica de São Paulo, com pósdoutorado em Estudos da Criança pelo Instituto da Criança da Universidade do Minho-Braga, Portugal, professor associado da Universidade Federal de Juiz de Fora, Brasil. Bolsista de Produtividade CNPq.

Telmo Mota Ronzani: doutor em Ciências da Saúde pela Universidade Federal de São Paulo, com pós-doutorado na área de álcool e outras drogas pela Universidade de São Paulo e estágio pós-doutoral pela University of Connecticut Health Center (UCHC), professor adjunto da Universidade Federal de Juiz de Fora, Brasil. Bolsista de Produtividade CNPq. 